\title{
Wnt5a induces ROR1 to associate with $14-3-3 \zeta$ for enhanced chemotaxis and proliferation of chronic lymphocytic
} leukemia cells

\author{
J Yu ${ }^{1,3}$, L Chen ${ }^{1,3}$, Y Chen ${ }^{1}$, MK Hasan ${ }^{1}$, EM Ghia ${ }^{1}$, L Zhang ${ }^{1}$, R Wu ${ }^{1}$, LZ Rassenti ${ }^{1}$, GF Widhopf ${ }^{1}$, Z Shen $^{2}$, SP Briggs ${ }^{2}$ and TJ Kipps $^{1}$
}

Wnt5a can activate Rho GTPases in chronic lymphocytic leukemia (CLL) cells by inducing the recruitment of ARHGEF2 to ROR1. Mass spectrometry on immune precipitates of Wnt5a-activated ROR1 identified 14-3-3ろ, which was confirmed by co-immunoprecipitation. The capacity of Wnt5a to induce ROR1 to complex with 14-3-3 could be blocked in CLL cells by treatment with cirmtuzumab, a humanized mAb targeting ROR1. Silencing 14-3-3 3 via small interfering RNA impaired the capacity of Wnt5a to: (1) induce recruitment of ARHGEF2 to ROR1, (2) enhance in vitro exchange activity of ARHGEF2 and (3) induce activation of RhoA and Rac1 in CLL cells. Furthermore, CRISPR/Cas9 deletion of 14-3-3 in ROR1-negative CLL cell-line MEC1, and in MEC1 cells transfected to express ROR1 (MEC1ROR1), demonstrated that 14-3-3 3 was necessary for the growth/engraftment advantage of MEC1-ROR1 over MEC1 cells. We identified a binding motif (RSPS ${ }^{857}$ SAS) in ROR1 for 14-3-3乙. Site-directed mutagenesis of ROR1 demonstrated that serine-857 was required for the recruitment of 14-3-3 3 and ARHGEF2 to ROR1, and activation of RhoA and Rac1. Collectively, this study reveals that 14-3-3 3 plays a critical role in Wnt5a/ROR1 signaling, leading to enhanced CLL migration and proliferation.

Leukemia (2017) 31, 2608-2614; doi:10.1038/leu.2017.132

\section{INTRODUCTION}

ROR1 is a developmentally restricted, type I tyrosine kinase-like orphan receptor expressed on the neoplastic cells of a variety of different cancers, ${ }^{1}$ including chronic lymphocytic leukemia (CLL), but not on most normal post-partum tissues. ${ }^{2}$ ROR1 is a receptor for Wnt5a, which can enhance the survival and growth of CLL cells. ${ }^{3}$ Furthermore, MEC1 cells made to express ROR1 (MEC1ROR1) had enhanced migration and growth compared with parental MEC1 cells, which express Wnt5a but lack expression of ROR1. ${ }^{1}$ Studies indicate that ROR1 may complex with a known co-activator of AKT, namely TCL1, ${ }^{3}$ and accelerate the development and progression of leukemia in $\mathrm{E} \mu-\mathrm{TCL} 1$ transgenic mice. ${ }^{3}$ Moreover, high-level leukemia cell expression of ROR1 is associated with accelerated disease progression in patients with CLL. ${ }^{4}$ On the other hand, silencing ROR1 in CLL cells can decrease leukemia cell survival. ${ }^{5}$ These studies imply that ROR1 signaling can promote leukemia cell activation and survival, and enhance disease progression in patients with CLL.

Studies indicated that Wnt5a-induced ROR1-dependent activation of Rho GTPases, RhoA and Rac1, by recruiting guanineexchange factors (GEFs), such as ARFGEF2. ${ }^{6}$ However, ARFGEF2 lacks a $\mathrm{SH} 3$ domain, suggesting other proteins are necessary for ARFGEF2 to complex with ROR1. Defining what protein(s) are required for recruitment to ROR1 of GEFs, such as ARFGEF2, could help elucidate the mechanism(s), whereby ROR1 is involved in enhancing migration and proliferation to promote tumor progression. Here we provide evidence that ROR1 can recruit ARHGEF2 via the adapter protein 14-3-3 , a member of the 14-3-3 family of conserved proteins, which plays a critical role in cell signaling pathways leading to enhanced proliferation, adhesion and survival of a variety of different cancers. ${ }^{7-9}$ Moreover, $14-3-3 \zeta$ appears necessary for Wnt5a-induced activation of RhoA and Rac1 via ARFGEF2, and required for Wnt5a-enhanced ROR $1^{+}$leukemia-cell proliferation, migration, and engraftment.

\section{MATERIALS AND METHODS}

CLL specimens and experimental animals

Blood samples were collected from CLL patients at the University of California-San Diego Moores Cancer Center, who satisfied diagnostic and immunophenotypic criteria for common B-cell CLL, and who provided written, informed consent, in compliance with the Declaration of Helsinki and the Institutional Review Board of the University of California-San Diego (Institutional Review Board approval number 080918). Peripheral blood mononuclear cells were isolated as described. ${ }^{6}$ All experiments with mice were conducted in accordance with the guidelines of the National Institutes of Health for the care and use of laboratory animals, and the University of California-San Diego approved the study protocol.

\section{Adoptive transfer in immune-deficient mice}

We injected $5 \times 10^{6}$ MEC1, MEC1- $14-3-3 \zeta$, MEC1-ROR1 or MEC1-ROR1$\triangle 14-3-3 \zeta$ cells into 6- to 8-week-old Rag2 ${ }^{-/-} \gamma_{c}^{-/-}$mice $(n=6)$ intravenously. All mice were killed for necropsy 2 weeks later and single-cell suspensions of splenocytes were purged of red blood cells by hypotonic lysis in ammonium-chloride-potassium lysis solution, washed, suspended in $2 \%(w t / v o l)$ BSA (Sigma-Aldrich, St Louis, MO, USA) in PBS $(\mathrm{pH}=7.4)$ and stained for surface expression of ROR1 (4A5) and CD19 using optimized concentrations of fluorochrome-conjugated mAbs. Cells were examined by four-color, multiparameter flow cytometry using a dual-laser FACSCalibur (BD Biosciences, San Jose, CA, USA) and the data were analyzed using FlowJo software (TreeStar, Ashland, OR, USA). The total number of

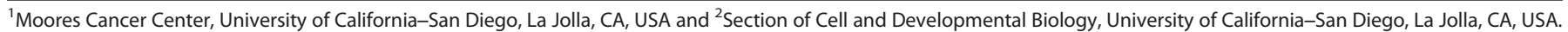
Correspondence: Dr TJ Kipps, Moores Cancer Center, University of California-San Diego, 3855 Health Sciences Drive, Room 4307, La Jolla CA 92093-0820, USA.

E-mail: tkipps@ucsd.edu

${ }^{3}$ These authors contributed equally to this work.

Received 30 November 2016; revised 3 March 2017; accepted 21 March 2017; accepted article preview online 3 May 2017; advance online publication, 16 June 2017 
leukemia cells per spleen was calculated by determining the percent of $\mathrm{CD} 19^{+} \mathrm{ROR} 1^{+}$cells of total lymphocytes by flow cytometry and multiplying this number by the total spleen cell count.

\section{RESULTS}

ROR1 complexes with 14-3-3द

We performed mass spectrometry-based proteomic analysis on antiROR1 immune precipitates of lysates prepared from freshly isolated CLL cells and identified 14-3-3 3 (Supplementary Figure S1A), a member of the 14-3-3 family of conserved adapter proteins. Immunoblot analysis of anti-ROR1 or anti-14-3-3 immune precipitates from CLL cell lysates confirmed that ROR1 can complex with 14-3-3Z (Figure 1a). Fluorescence confocal microscopy demonstrated that 14-3-3 Co-localized with ROR1 in freshly isolated CLL cells (Figures 1b and c). In contrast, control studies did not detect co-localization of 14-3-3 3 with an unrelated surface protein, CD5 (Supplementary Figure S1B and C). We incubated CLL cells with an anti-ROR1 antibody (cirmtuzumab) specific for a ROR1 epitope distinct from that recognized by $4 A 5$ and found cirmtuzumab could disrupt the ROR1-14-3-3Z complex (Figures $1 \mathrm{~b}$ and $\mathrm{c}$ ).

Wnt5a is a ligand for ROR1 that can induce ROR1-dependent signaling. Culture of CLL cells for $16 \mathrm{~h}$ in Wnt5a-deficient media resulted in the disintegration of the ROR1-14-3-3ろ complex, which was apparent in CLL cells that were freshly isolated from the blood. When we treated such cells with Wnt5a, we induced ROR1 to re-associate with 14-3-3 . Again, we noted that treatment of the CLL cells with cirmtuzumab inhibited the capacity of Wnt5a to induce recruitment of $14-3-3 \zeta$ to ROR1 (Figures $1 d-f$ ).

\section{4-3-3ろ interacts with ARHGEF2}

Prior studies demonstrated that $14-3-3 \zeta$ can interact with ARHGEF2, ${ }^{10}$ facilitating activation of RhoA and Rac1. ${ }^{6}$ We confirmed that 14-3-3Z complexes with ARHGEF2 in freshly isolated CLL cells via immunoprecipitation and immunoblot analyses (Figure 2a). We hypothesized that 14-3-3 3 was required for the recruitment and activation of ARHGEF2 to ROR1, allowing for activation of RhoA and Rac1 following Wnt5a stimulation. We silenced $14-3-3 \zeta$ with specific small interfering RNA (siRNA) in CLL cells, which were cultured with or without exogenous Wnt5a. Immunoprecipitation analysis indicated that 14-3$3 \zeta$-specific siRNA reduced the expression level of 14-3-3 3 and impaired the capacity of Wnt5a to induce ROR1 to associate with ARHGEF2 (Figure 2b). Fluorescence confocal microscopy showed that Wnt5a induced significantly greater co-localization of ROR1 with ARHGEF2 in CLL cells transfected with control siRNA than in the same

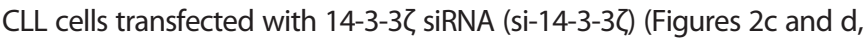
and Supplementary Figures S2A-D). Moreover, treatment of CLL cells with 14-3-3Z-specific siRNA, but not control siRNA, inhibited the capacity of Wnt5a to activate ARHGEF2, as assessed by the capacity of anti-ARHGEF2 immune precipitates to activate RhoA or Rac1 in vitro (Figure 2e and Supplementary Figures S2E and F). Moreover, Wnt5a was less effective in activating RhoA and Rac1 in CLL cells transfected with si-14-3-3 3 than in CLL cells transfected with control siRNA (Figure $2 f$ and Supplementary Figures S2E and F). These data imply that $14-3-3 \zeta$ was required for the recruitment to ROR1 and activation of ARHGEF2 in response to Wnt5a.

\section{4-3-3 3 enhances $\mathrm{ROR}{ }^{+}{ }^{+}$leukemia progression in vitro}

MEC1 cells were derived from CLL and have been used as a cell model for this leukemia. ${ }^{11}$ However, MEC1 cells lack expression of a

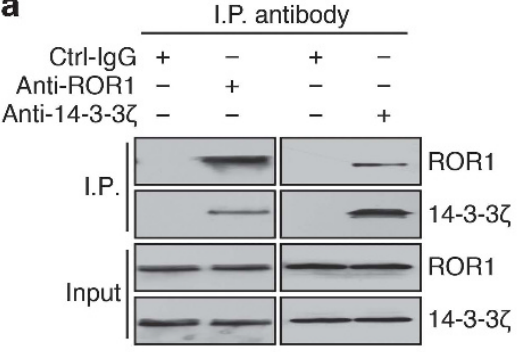

b

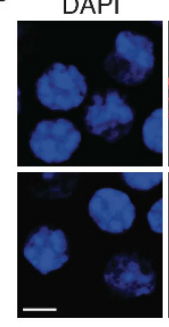

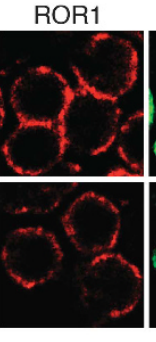
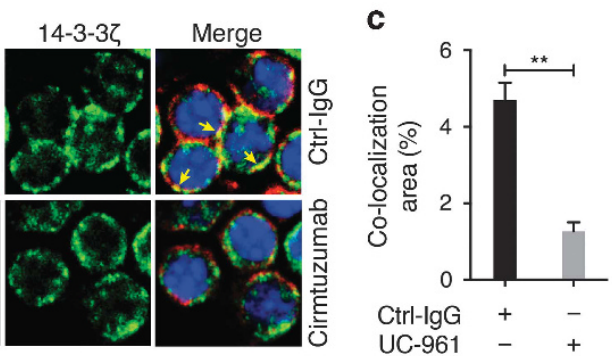

e

d

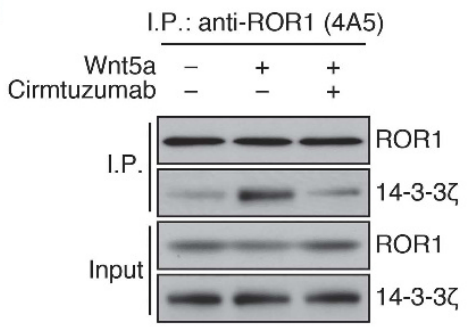

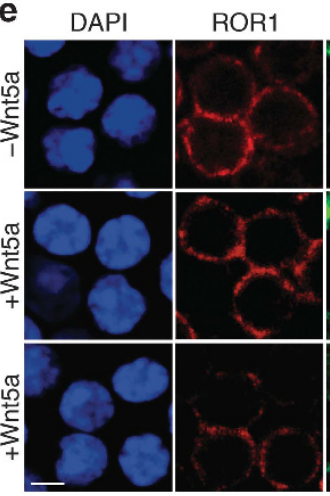

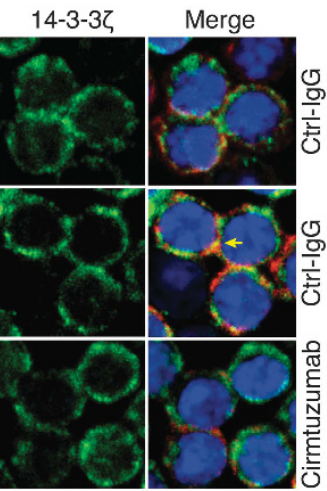

C

UC-961 - +

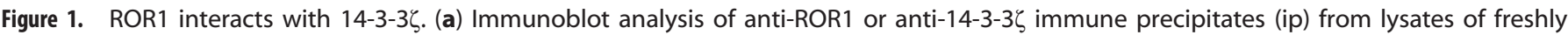

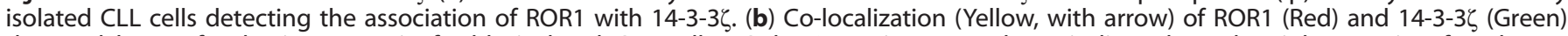
detected by confocal microscopy in freshly isolated CLL cells \pm Ctrl-lgG or cirmtuzumab, as indicated on the right margin of each row (Objective: $\times 100$; scale bar $=5 \mu \mathrm{m}$ ). (c) Percentage of co-localization area of ROR1 and $14-3-3 \zeta$ in freshly isolated CLL cells \pm Ctrl-lgG or cirmtuzumab $(n=3)$. Data are shown as mean \pm s.e.m.; ${ }^{* *} P<0.01$, as calculated using two-tailed Student's $t$-test. (d) Immunoblot analysis of anti-ROR1 ip from lysates of cultured CLL cells treated with or without cirmtuzumab, demonstrating the capacity of ROR1 to associate with

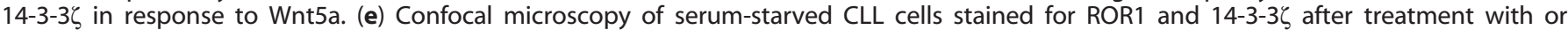
without Wnt5a and Ctrl-IgG or cirmtuzumab, as indicated on the right margin of each row (Objective: $\times 100 ;$ scale bar $=5 \mu \mathrm{m}$ ). (f) Percentage of the cell area in which we detect co-localization of ROR1 and 14-3-3 $\zeta$ by confocal microscopy in serum-starved CLL cells \pm Wnt5a and Ctrl-lgG or cirmtuzumab $(n=3)$. Data are shown as mean \pm s.e.m.; ${ }^{* *} P<0.01$, as calculated using one-way analysis of variance with Tukey's multiple comparisons test. 
a

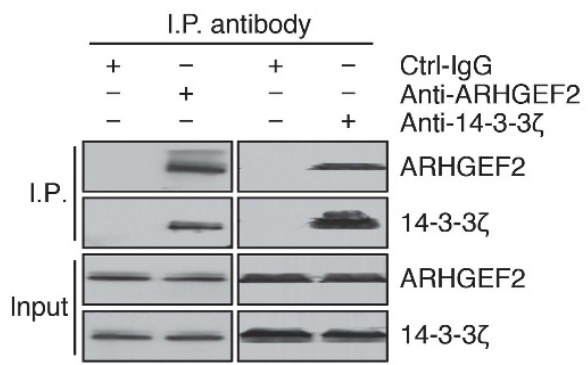

b

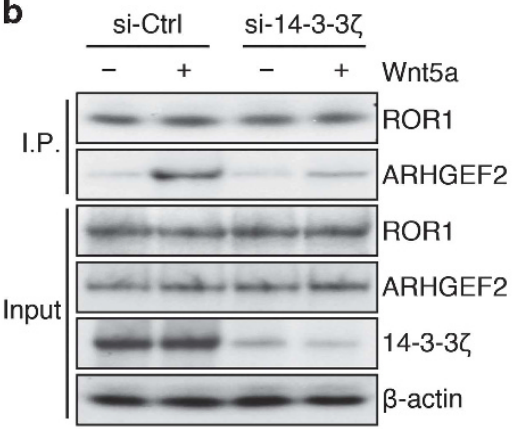

e
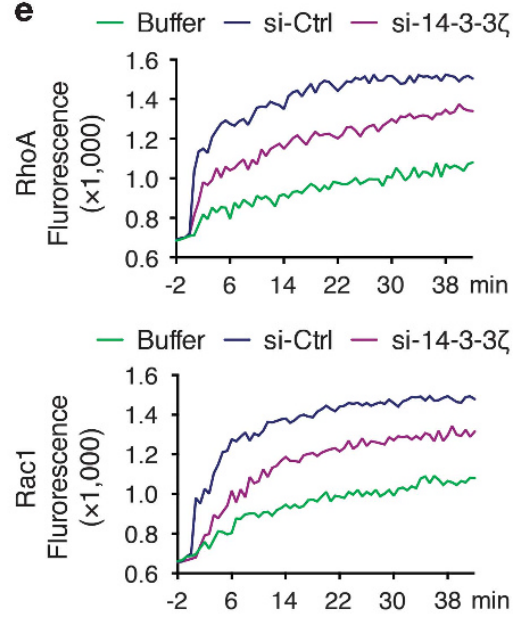

$\mathbf{f}$

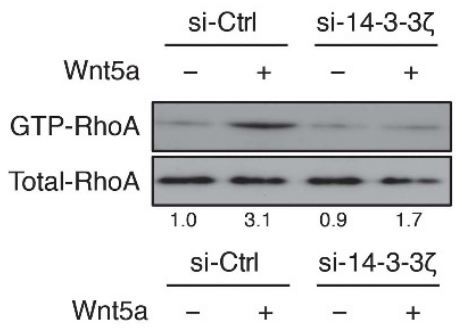

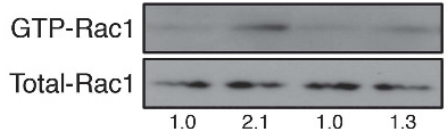

Figure 2. Interaction of 14-3-3 $\zeta$ with ARHGEF2. (a) Immunoblot analysis of immune precipitates (ip) generated using lysates of freshly isolated CLL cells with a mAb specific for 14-3-3 14-3-3 $\zeta$ or ARHGEF2 as indicated on the left margin of each subpanel. (b) Immunoblot analysis of anti-ROR1 ip from lysates of cultured CLL

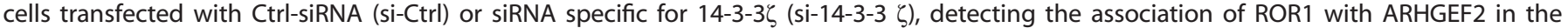
presence, but not in the absence, of Wnt5a. (c) Co-localization (yellow, with arrow) of ROR1 (red) and ARHGEF2 (green) detected by confocal

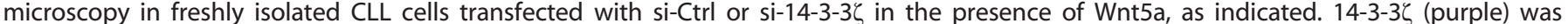
stained to confirm the silencing efficiency (Objective: $\times 100$; scale bar $=5 \mu \mathrm{m}$ ). (d) Percentage of co-localization area of $14-3-3 \zeta$ and ARHGEF2 detected by confocal microscopy in serum-starved CLL cells transfected with Ctrl-siRNA or siRNA specific for YWHAZ, encoding $14-3-3 \zeta$, in the presence or absence of Wnt5a, as indicated $(n=3)$. Data are shown as mean \pm s.e.m.; ${ }^{*} P<0.05$, as calculated using one-way analysis of variance with Tukey's multiple comparisons test. (e) In vitro exchange assay on RhoA or Rac1 of ip from lysates of CLL cells

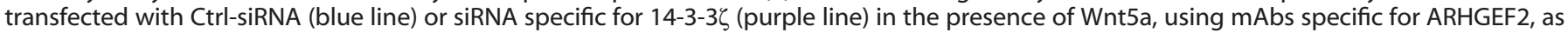
indicated in the bottom of each graph. The green line depicts GTPase activation using buffer alone. (f) Activation of RhoA or Rac1 following treatment without (-) or with (+) Wnt5a of CLL cells transfected with Ctrl-siRNA or siRNA specific for 14-3-36. Whole-cell lysates also were examined via immunoblot analysis for total RhoA or Rac1. The number beneath each lane is the ratio of band densities for activated versus total GTPase normalized for untreated samples.

ROR1 (Supplementary Figure S3A), but express Wnt5a. ${ }^{6}$ We made stable transfectants of MEC1 that expressed full-length ROR1 to generate MEC1-ROR1 cells and found that MEC1-ROR1 cells had higher rates of chemokine-induced migration and proliferation than parental MEC1 cells. ${ }^{1,6}$

We performed mass spectrometry on anti-ROR 1 immune precipitates from MEC1-ROR1 cell lysates and also detected 14-3-37 (Supplementary Figure S3B). To examine the function of 14-3-3Z, we extinguished expression of $Y W H A Z$, the gene encoding 14-3-3Z, in MEC1 or MEC1-ROR1 cells by CRISPR/Cas9 technology (Supplementary Figures S3C and D). We found that MEC1-ROR1 cells had higher rates of cell proliferation than MEC1 or MEC1ROR1- $\triangle 14-3-3 \zeta$ cells, which proliferated at rates comparable to that of MEC1- $\Delta 14-3-3 \zeta$, which lacked the expression of both ROR1 and 14-3-3 (Figure 3a). MEC1-ROR1 cells also had higher rates of cell migration in response to CCL21 than MEC1 or MEC1-ROR1$\triangle 14-3-3 \zeta$ cells, which again migrated at rates comparable to that of MEC1- $\Delta 14-3-3 \zeta$ lacking expression of both ROR1 and 14-3-3 (Figure 3b). Immunoprecipitation studies revealed that ROR1 interacts with ARHGEF2 in MEC1-ROR1 cells, but not in MEC1ROR1- $\Delta 14-3-3 \zeta$ cells (Figure $3 c$ ). In addition, MEC1-ROR1 cells had higher levels of activated Rac1 and RhoA than did MEC1 cells or MEC1-ROR1- $\Delta 14-3-3 \zeta$ cells (Figure $3 d$ ). These data indicate that $14-3-3 \zeta$ is necessary for the recruitment and activation of ARHGEF2 and the proliferation and migration advantage of MEC1-ROR1 over MEC1 cells in vitro.

\section{4-3-3 enhances $\mathrm{ROR} 1^{+}$leukemia progression in vivo}

We examined the capacity of MEC1-ROR1, MEC1, MEC1- $\Delta 14-3-3 \zeta$ or MEC1-ROR1- $\Delta 14-3-3 \zeta$ cells to engraft immune-deficient mice. Two weeks after intravenous infusion of equal numbers of cells, Rag $2^{-1-} \gamma_{c}^{-1-}$ mice engrafted with $\mathrm{CD} 19^{+} / \mathrm{ROR}^{+}$MEC1-ROR1 cells had significantly greater splenomegaly than did littermates engrafted with MEC1, MEC1- $\Delta 14-3-3 \zeta$ or MEC1-ROR1- $14-3-3 \zeta$ cells (Supplementary Figure S3E). The proportion of human CD19 ${ }^{+}$leukemia cells in each spleen was determined by flow cytometric analysis of isolated splenocytes (Figure 4a). Multiplying this number by the total number of splenocytes allowed us to 


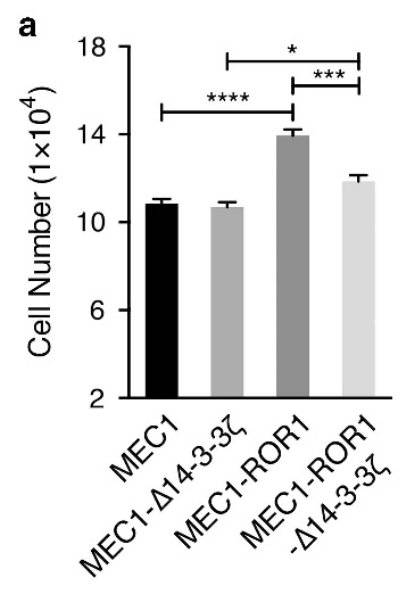

b

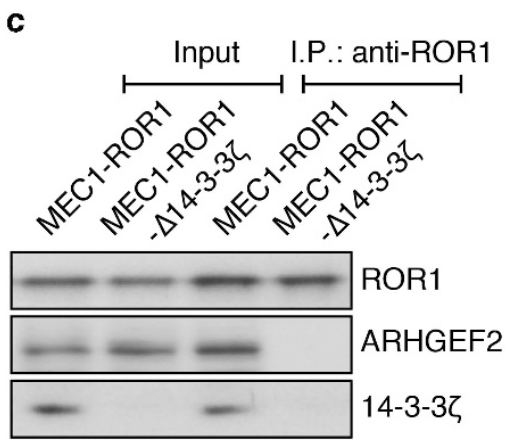

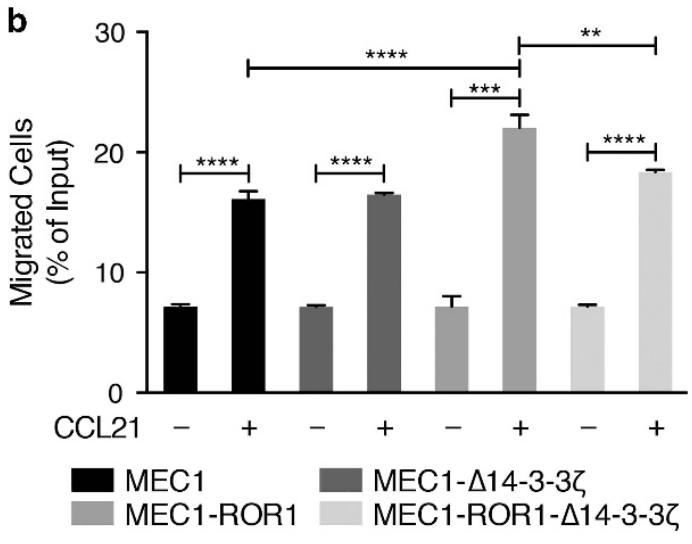

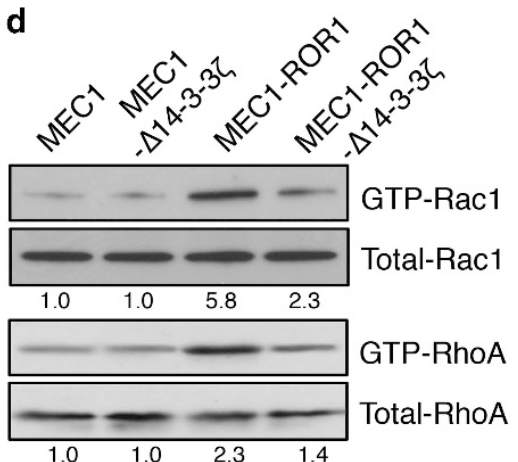

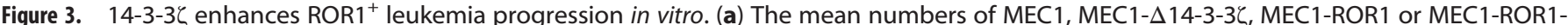
$\Delta 14-3-3 \zeta$ cells in triplicate wells at the days indicated below the graph. (b) The bars indicate the mean proportions of MEC1, MEC1- $\Delta 14-3-3 \zeta$, MEC1-ROR1 or MEC1-ROR1- $\Delta 14-3-3 \zeta$ cells migrating with $(+)$ or without $(-)$ CCL21, as indicated at the bottom ( $n=3)$. (c) Interaction of ROR1 with ARHGEF2 was confirmed by immunoblot analysis of anti-ROR1 immune precipitates (ip) using lysates from MEC1-ROR1 or MEC1ROR1- $\Delta$ 14-3-3C cells. (d) Activated Rac1 or RhoA were measured in MEC1, MEC1- 14 -3-3C, MEC1-ROR1 or MEC1-ROR1- $14-3-3 \zeta$ cells. Wholecell lysates also were examined via immunoblot for total Rac1 or RhoA. The numbers beneath each lane are the ratios of band densities for activated versus total GTPase normalized for sample of MEC1 cells. Data are shown as mean \pm s.e.m.; ${ }^{*} P<0.05,{ }^{* *} P<0.01,{ }^{* * *} P<0.001$ and ****P $<0.0001$, as calculated using one-way analysis of variance with Tukey's multiple comparisons test.

a
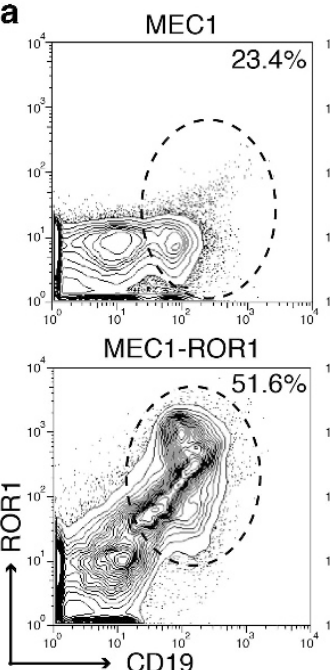
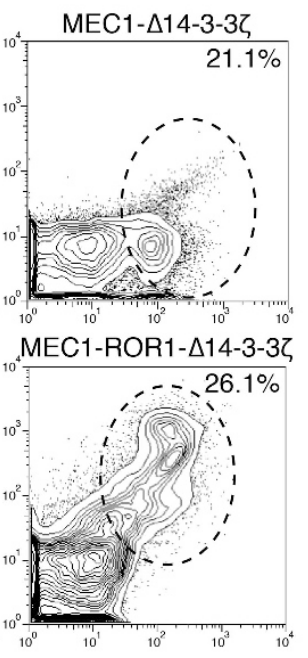

b

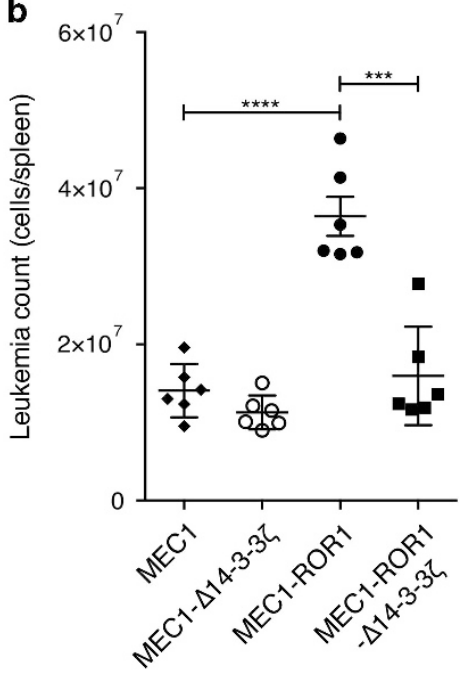

Figure 4. 14-3-3ל enhances $\mathrm{ROR} 1^{+}$leukemia progression in vivo. (a) Leukemia cells were collected from the spleens of engrafted mice. The fluorescence of cells stained with 4A5-Alexa-647 (ordinate) and anti-CD19-PE (abscissa) are shown in the contour plots. The percentages at the top right of each contour plot indicate the proportions of cells with fluorescence above the threshold indicated by the dotted circle. (b) The bars indicate the average numbers of CD19+ROR $1^{+}$human leukemia cells harvested from spleens of mice engrafted 2 weeks earlier with MEC1, MEC1- $\Delta 14-3-3 \zeta$, MEC1-ROR1 or MEC1-ROR1- $\Delta 14-3-3 \zeta$ cells, as indicated at the bottom of each histogram $(n=6)$. Data are shown as mean \pm s.e.m.; ${ }^{* * *} P<0.001$ and ${ }^{* * *} P<0.0001$, as calculated using one-way analysis of variance with Tukey's multiple comparisons test. 


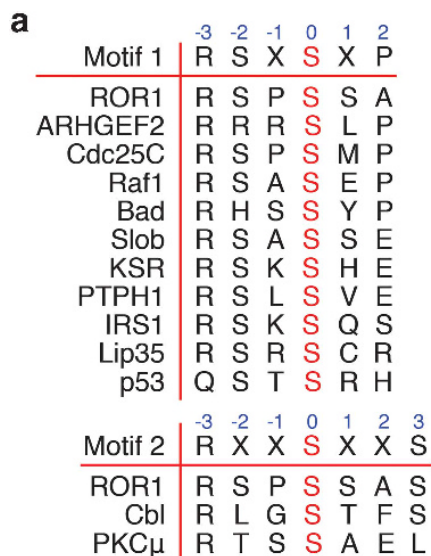

\begin{tabular}{|c|c|c|c|c|c|}
\hline $\begin{array}{l}\text { Motif } 1 \\
\text { Motif } 2\end{array}$ & & $\begin{array}{l}\mathrm{R} S \mathrm{~S} \\
\mathrm{R} X \mathrm{X}\end{array}$ & & & \\
\hline Human & 854 & 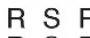 & $P$ & & \\
\hline himpanzee & 854 & R S & & & \\
\hline Bovine & 825 & R S F & & & \\
\hline Cattle & 854 & R S & & & \\
\hline Hourse & 814 & R S & & & \\
\hline Pig & 836 & R S & & & \\
\hline Dog & 791 & R S & & & \\
\hline Rabbit & 854 & $R S$ & & & \\
\hline Mouse & 854 & R S P & & & \\
\hline
\end{tabular}
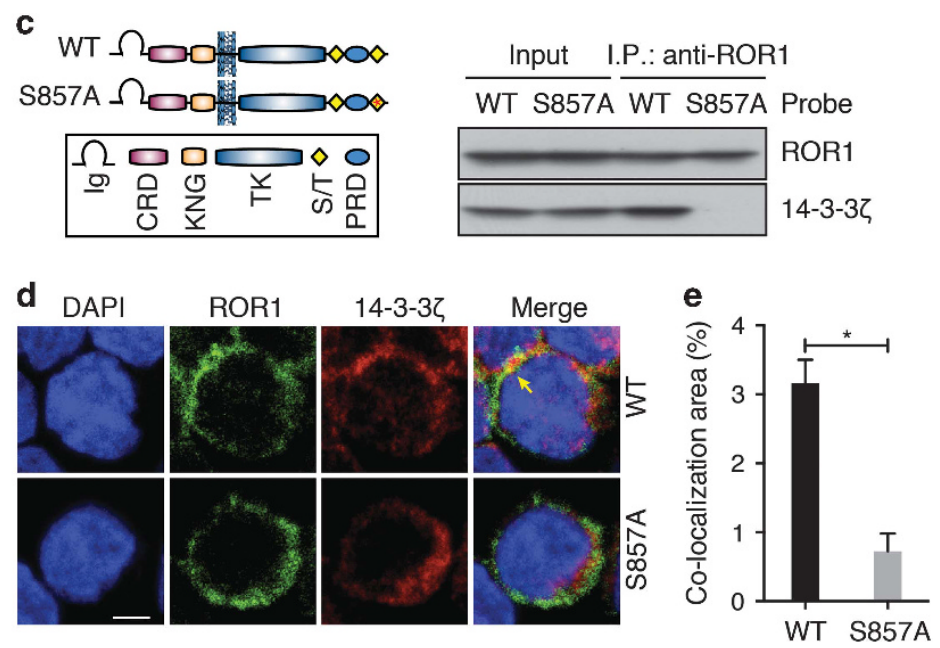

\section{f}
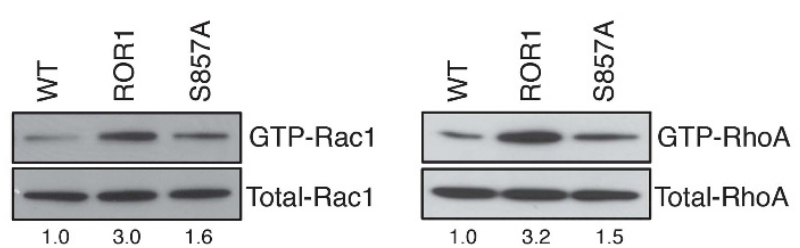

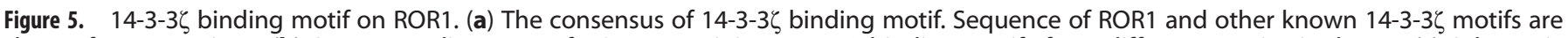
shown for comparison. (b) Sequence alignment of ROR1 containing 14-3-3C binding motifs from different species is shown. (c) Schematic representation of the structure of ROR1 and mutated form of ROR1 (ROR1-S857A) was shown. Interaction of ROR1 with 14-3-3 $\zeta$ was confirmed by immunoblot analysis of anti-ROR1 immune precipitates (ip) using lysates from MEC1-ROR1 (WT) or MEC1 cells transfected with ROR1S857A (S857A). (d) Co-localization (yellow, with arrow) of ROR1 (green) with 14-3-3ל (red) in MEC1-ROR1 and MEC1-ROR1-S857A cells, as indicated on the right margin of each row (objective: $\times 100$; scale bar: $2 \mu \mathrm{m}$ ). (e) Percentage of cell area in which we detected co-localization of ROR1 and 14-3-3 $\zeta$ in MEC1-ROR1 or MEC1-ROR1-S857A cells $(n=3)$. Data are shown as mean \pm s.e.m.; ${ }^{*} P<0.05$, as determined by two-tailed Student's $t$ test. (f) Activated Rac1 or RhoA were measured in MEC1 (WT), MEC1-ROR1 (ROR1) or ROR1-S857A cells (S857A). Whole-cell lysates also were examined via immunoblot for total Rac1 or RhoA. The numbers beneath each lane are the ratios of band densities for activated versus total GTPase normalized with respect to that of MEC1 cells.

calculate the total number of $\mathrm{CD} 19^{+}$leukemia cells per spleen for each mouse (Figure $4 \mathrm{~b}$ ). This revealed that Rag $2^{-/-} \gamma_{c}^{-1-}$ mice engrafted with MEC1-ROR1 had significantly greater numbers of leukemia cells than did littermates engrafted with MEC1, MEC1$\Delta 14-3-3 \zeta$ or MEC1-ROR1- $\Delta 14-3-3 \zeta$ cells (Figure $4 b$ ).

The gene expression profiling of CLL samples revealed variable expression levels of YWHAZ mRNA; the median level of YWHAZ mRNA in CLL cells that expressed unmutated immunoglobulin heavy-chain variable region genes (IGHV) (UM-CLL) was significantly higher than that observed in CLL cells that used mutated IGHV (M-CLL) (Supplementary Figure S4A). To validate these findings, we selected eight UM-CLL cell samples and eight M-CLL cell samples, and examined for expression of YWHAZ cDNA by quantitative reverse transcriptase-PCR (Supplementary Figure S4B) and for 14-3-3 3 protein by immunoblot analysis (Supplementary Figure S4C). Again, we found YWHAZ expressed in all samples tested; however, we found significantly higher levels of YMHAZ in UM-CLL than in M-CLL (Supplementary Figure S4B). We also found significantly higher levels of $14-3-3 \zeta$ in UM-CLL than in M-CLL by immunoblot analysis (Supplementary Figures S4C and D). The relative levels of YWHAZ CDNA detected by quantitative reverse transcriptase-PCR correlated with the relative levels of $14-3-3 \zeta$ detected by immunoblot analysis $\left(R^{2}=0.9254\right.$, Supplementary Figure S4E).

\section{Serine-857 of ROR1 is required for $14-3-3 \zeta$ binding}

Using methods to predict 14-3-3ろ-binding peptides, for example, 14-3-3-Pred, ${ }^{12}$ we predicted that the serine-857 (RSPS ${ }^{857}$ SA) of ROR1 was a potential $14-3-3 \zeta$ binding site, as $14-3-3 \zeta$ preferentially binds to target proteins with two consensus RSXpSXP and $\mathrm{RX}_{1-2} \mathrm{pSX}_{2-3} \mathrm{~S}$ motifs. ${ }^{13,14}$ Moreover, we found that the predicted
14-3-3 binding motifs in the cytoplasmic domain of ROR1 were similar to those of other known 14-3-3 substrates (Figure 5a); such motifs are evolutionarily conserved in mammals (Figure $5 b$ ). Although studies by other investigators found phosphorylation of the serines in the intracellular domain of ROR1 in CLL, ${ }^{15}$ we could not verify that serine at position 857 undergoes changes in phosphorylation from our mass spectrometry data. In addition, as there is no antibody specific for the phospho-Serine-857 of ROR1, we could not verify that serine 857 was phosphorylated via immunoblot analysis. For this reason, we generated a mutant form of ROR1 that had a serine-to-alanine substitution at position 857 . Immunoprecipitation studies revealed that $14-3-3 \zeta$ interacts with ROR1 in MEC1-ROR1 cells, expressing wild-type ROR1, but not in MEC1-ROR1 ${ }^{\text {S857A }}$ cells (Figure 5c). Fluorescence confocal microscopy demonstrated that ROR1 and 14-3-3 3 co-localized in MEC1-ROR1 cells; however, we did not observe co-localization of ROR1 with $14-3-3 \zeta$ in MEC1-ROR1 ${ }^{\text {S857A }}$ cells (Figures $5 \mathrm{~d}$ and e). Accordingly, MEC1-ROR1 cells had higher levels of activated Rac1 and RhoA than MEC1 or MEC1-ROR $1^{\text {S857A }}$ cells (Figure 5f). These data imply that Serine-857 of ROR1 is required for the interaction of $14-3-3 \zeta$ with ROR1, leading to activation of ARHGEF2.

\section{DISCUSSION}

In the present study, we provide evidence that $14-3-3 \zeta$ was necessary for the recruitment of ARHGEF2 to ROR1 and the activation of the exchange activity of ARHGEF2 for RhoA and Rac1 in response to $W n t 5 a$. First, we validated the Wnt5a-induced interaction of ROR1 with $14-3-3 \zeta$ in primary CLL cells using coimmunoprecipitation studies and immunoblot analyses. Next, we 
demonstrated that expression of $14-3-3 \zeta$ was necessary for Wnt5aenhanced chemokine-directed migration and proliferation of primary CLL cells in vitro and MEC1-ROR1 cell engraftment ability in vivo. Furthermore, the effect of $14-3-3 \zeta$ was dependent on expression of ROR1. Thus, the present study identifies a novel function of $14-3-3 \zeta$ as an adaptor protein that can participate in the differential regulation of Rac1 or RhoA-induced changes in cell behavior by localizing a GEF to ROR1 to facilitate Rac1 or RhoAinduced signaling.

14-3-3 ל belongs to a family of scaffold proteins that are involved in many important cellular functions and that regulate multiple signal transduction pathways. ${ }^{16,17}$ 14-3-3 proteins are adaptor proteins that bind to a variety of cellular targets, including the proapoptotic protein Bad, Raf kinase, cell cycle-dependent phosphatase $\mathrm{Cdc} 25$ and others. ${ }^{18}$ Thus, 14-3-3 binding may regulate the activity of these proteins and their ability to interact with other components of intracellular signaling pathways.

14-3-3 proteins have been defined as phosphoserine-binding proteins, which bind to the consensus motif RSXPSXP. ${ }^{13}$ Two different binding motifs have been identified: RSXpSXP (mode I) and RXY/FXpSXP (mode II). ${ }^{7,13,19}$ In addition to these two canonical binding motifs, 14-3-3 can bind to the extreme $\mathrm{COOH}$ terminus of several proteins, recently defined as a mode III binding site. ${ }^{20,21}$ Studies have identified another 14-3-3-binding motif in Cbl, namely $\mathrm{RX}_{1-2} \mathrm{SX}_{2-3} \mathrm{~S}_{1}^{14}$ which has been found in Keratin $18^{22}$ and PKC $\mu .{ }^{23}$ After analyzing the sequence of ROR1 for potential 14-3-3 binding sites, we identified a motif, RSPSSAS, which was located in the serine/threonine-rich domain at positions 854-860 of ROR1 that was consistent with both mode I and Cbl-like motifs. Although we could not verify that serine-857 in this motif was phosphorylated in CLL cells or that $14-3-3 \zeta$ directly bound to this ROR1 motif in vivo, our studies demonstrate that substitution of alanine for serine at position 857 effectively abrogated the capacity of ROR1 to complex with 14-3-3द.

The present findings also extended our understanding of the importance of 14-3-3 in ROR1-dependent Wnt5a-induced signaling. We found that reducing expression of $14-3-3 \zeta$ via RNA interference impaired the capacity of Wnt5a to: (1) induce recruitment of ARHGEF2 to ROR1, (2) enhance the in vitro exchange activity of ARHGEF2 for RhoA and Rac1, and (3) induce the activation of RhoA and Rac1 in primary CLL cells. In addition, MEC1-ROR1 had significantly higher rates of proliferation or migration than MEC1-ROR1- $\Delta 14-3-3 \zeta$ cells, which in turn had rates of proliferation or migration comparable to those of MEC1 cells or MEC1- $\Delta 14-3-3 \zeta$ lacking expression of ROR1. Moreover, we found that $14-3-3 \zeta$ was required for the engraftment/growth advantage of MEC1-ROR1 over MEC1 cells in vivo. Although we found 14-3-37 expressed in each CLL sample examined, we found higher levels in CLL cells that use UM-IGHV than in CLL cells that express M-IVHG, prompting us to speculate that the relative level of 14-3-37 contributes to the noted aggressive clinical behavior of UM-CLL relative to that of $\mathrm{M}-\mathrm{CLL} .{ }^{24}$ In any case, we conclude that $14-3-3 \zeta$ is the isoform of 14-3-3 that has a key role in ROR1-dependent Wnt5a signaling in CLL.

Prior studies noted that $14-3-3 \zeta$ also could mediate integrininduced activation of cdc42 and Rac1. ${ }^{25}$ Moreover, 14-3-3 3 may interact with ARHGEF2 and help regulate ARHGEF2 activity, which can induce Rho- and Rac-mediated signaling. ${ }^{26}$ Similar to our findings, $14-3-3 \zeta$ was reported to mediate the recruitment of the Rac1-GEF, Tiam1, to $\beta 1$-integrin complexes, which also resulted in activation of Rac1. ${ }^{27}$ Unlike Tiam1, which only is expressed by activated lymphocytes and activated CLL cells, and appears unable to enhance mobility of CLL cells, ${ }^{28}$ ARHGEF2 is expressed by resting lymphocytes and can promote CLL proliferation and migration. 6,29

In summary, the present study describes a previously unrecognized ROR1/14-3-3乙/ARHGEF2-dependent mechanism for generating active Rac1 or RhoA in Wnt5a/ROR1 signal that contributes to CLL proliferative or motile behaviors. The findings reported here demonstrate the importance of 14-3-3Z in ROR1-dependent Wnt5a-induced signaling and highlight a pathway for potential drug development. To this end, we found that the capacity of Wnt5a to induce ROR1 to associate with 14-3-3ろ could be blocked by cirmtuzumab, a first-in-class humanized mAb specific for a functional epitope in the ROR1-extracellular domain that is being evaluated in patients with CLL (https://clinicaltrials.gov/ct2/show/ NCT02222688). ${ }^{30}$ Moreover, we found that cirmtuzumab could block the capacity of Wnt5a to induce recruitment of 14-3-3Z to ROR1 and activation of ARHGEF2 contributing to the noted capacity of cirmtuzumab to block ROR1-dependent non-canonical Wnt5a signaling responsible for enhanced leukemia-cell proliferation. As such, these studies support the rationale for clinical evaluation of this antibody in patients with CLL.

\section{CONFLICT OF INTEREST}

Cirmtuzumab was developed in the Kipps laboratory and licensed by Oncternal Therapeutics, Inc., from the University of California, San Diego.

\section{ACKNOWLEDGEMENTS}

We thank Dr Kersi Pestonjamasp for fluorescence confocal microscopy analysis and University of California-San Diego Moores Cancer Center Microscopy Core Facility for technical help. We thank California Institute for Regenerative Medicine (CIRM) grant (DR3-06924) for supporting us to generate anti-ROR1 mAb, cirmtuzumab. This work was supported by the UC San Diego Foundation Blood Cancer Research Fund (BCRF), a SPORE grant (7005-14) from the Leukemia and Lymphoma Society, and a PO1 grant (5P01CA081534-14) from the NIH for the CLL Research Consortium.

\section{AUTHOR CONTRIBUTIONS}

JY and TJK conceived the project. JY, LC, YC, EMG, LJZ, ZS, LZ and RW performed the experiments. JY, LC, YC, EMG, LZR, GFW, ZS, SPB and TJK analyzed data. JY, LC and TJK wrote the manuscript.

\section{REFERENCES}

1 Zhang S, Chen L, Wang-Rodriguez J, Zhang L, Cui B, Frankel W et al. The oncoembryonic antigen ROR1 is expressed by a variety of human cancers. Am J Pathol 2012; 181: 1903-1910.

2 Fukuda T, Chen L, Endo T, Tang L, Lu D, Castro JE et al. Antisera induced by infusions of autologous Ad-CD154-leukemia B cells identify ROR1 as an oncofetal antigen and receptor for Wnt5a. Proc Natl Acad Sci USA 2008; 105: 3047-3052.

3 Widhopf GF 2nd, Cui B, Ghia EM, Chen L, Messer K, Shen Z et al. ROR1 can interact with TCL1 and enhance leukemogenesis in Emu-TCL1 transgenic mice. Proc Natl Acad Sci USA 2014; 111: 793-798.

4 Cui B, Ghia EM, Chen L, Rassenti LZ, DeBoever C, Widhopf GF 2nd et al. High-level ROR1 associates with accelerated disease-progression in chronic lymphocytic leukemia. Blood 2016; 128: 2931-2940.

5 Choudhury A, Derkow K, Daneshmanesh AH, Mikaelsson E, Kiaii S, Kokhaei P et al. Silencing of ROR1 and FMOD with siRNA results in apoptosis of CLL cells. $\mathrm{Br} J$ Haematol 2010; 151: 327-335.

6 Yu J, Chen L, Cui B, Widhopf GF 2nd, Shen Z, Wu R et al. Wnt5a induces ROR1/ ROR2 heterooligomerization to enhance leukemia chemotaxis and proliferation. J Clin Invest 2016; 126: 585-598.

7 Hermeking $\mathrm{H}$. The 14-3-3 cancer connection. Nat Rev Cancer 2003; 3: 931-943.

8 Matta A, Siu KW, Ralhan R. 14-3-3 zeta as novel molecular target for cancer therapy. Expert Opin Ther Targets 2012; 16: 515-523.

9 Neal CL, Yu D. 14-3-3zeta as a prognostic marker and therapeutic target for cancer. Expert Opin Ther Targets 2010; 14: 1343-1354.

10 Jin J, Smith FD, Stark C, Wells CD, Fawcett JP, Kulkarni S et al. Proteomic, functional, and domain-based analysis of in vivo 14-3-3 binding proteins involved in cytoskeletal regulation and cellular organization. Curr Biol 2004; 14: 1436-1450.

11 Stacchini A, Aragno M, Vallario A, Alfarano A, Circosta P, Gottardi D et al. MEC1 and MEC2: two new cell lines derived from B-chronic lymphocytic leukaemia in prolymphocytoid transformation. Leukemia Res 1999; 23: 127-136.

12 Madeira F, Tinti M, Murugesan G, Berrett E, Stafford M, Toth R et al. 14-3-3-Pred: improved methods to predict 14-3-3-binding phosphopeptides. Bioinformatics 2015; 31: 2276-2283. 
13 Muslin AJ, Tanner JW, Allen PM, Shaw AS. Interaction of 14-3-3 with signaling proteins is mediated by the recognition of phosphoserine. Cell 1996; 84: 889-897.

14 Liu YC, Liu Y, Elly C, Yoshida H, Lipkowitz S, Altman A. Serine phosphorylation of $\mathrm{Cbl}$ induced by phorbol ester enhances its association with 14-3-3 proteins in T cells via a novel serine-rich 14-3-3-binding motif. J Biol Chem 1997; 272: 9979-9985.

15 Hojjat-Farsangi M, Khan AS, Daneshmanesh AH, Moshfegh A, Sandin A, Mansouri $L$ et al. The tyrosine kinase receptor ROR1 is constitutively phosphorylated in chronic lymphocytic leukemia (CLL) cells. PLoS One 2013; 8: e78339.

16 Zhang J, Chen F, Li W, Xiong Q, Yang M, Zheng P et al. 14-3-3zeta interacts with stat 3 and regulates its constitutive activation in multiple myeloma cells. PLoS One 2012; 7: e29554.

17 Xu J, Acharya S, Sahin O, Zhang Q, Saito Y, Yao J et al. 14-3-3zeta turns TGF-beta's function from tumor suppressor to metastasis promoter in breast cancer by contextual changes of Smad partners from p53 to Gli2. Cancer Cell 2015; 27: 177-192.

$18 \mathrm{Fu} \mathrm{H}$, Subramanian RR, Masters SC. 14-3-3 proteins: structure, function, and regulation. Annu Rev Pharmacol Toxicol 2000; 40: 617-647.

19 Yaffe MB, Rittinger K, Volinia S, Caron PR, Aitken A, Leffers $\mathrm{H}$ et al. The structural basis for 14-3-3:phosphopeptide binding specificity. Cell 1997; 91: 961-971.

20 Coblitz B, Shikano S, Wu M, Gabelli SB, Cockrell LM, Spieker M et al. C-terminal recognition by $14-3-3$ proteins for surface expression of membrane receptors. J Biol Chem 2005; 280: 36263-36272.

21 Shikano S, Coblitz B, Sun H, Li M. Genetic isolation of transport signals directing cell surface expression. Nat Cell Biol 2005; 7: 985-992.

$22 \mathrm{Ku}$ NO, Liao J, Omary MB. Phosphorylation of human keratin 18 serine 33 regulates binding to 14-3-3 proteins. EMBO J 1998; 17: 1892-1906.

23 Hausser A, Storz P, Link G, Stoll H, Liu YC, Altman A et al. Protein kinase C mu is negatively regulated by 14-3-3 signal transduction proteins. J Biol Chem 1999; 274: 9258-9264.

24 Kipps TJ, Stevenson FK, Wu CJ, Croce CM, Packham G, Wierda WG et al. Chronic lymphocytic leukaemia. Nat Rev Dis Primers 2017; 3: 17008-17028.
25 Bialkowska K, Zaffran Y, Meyer SC, Fox JE. 14-3-3 zeta mediates integrininduced activation of $\mathrm{Cdc} 42$ and Rac. Platelet glycoprotein Ib-IX regulates integrin-induced signaling by sequestering 14-3-3 zeta. J Biol Chem 2003; 278: 33342-33350.

26 Zenke FT, Krendel M, DerMardirossian C, King CC, Bohl BP, Bokoch GM. p21-activated kinase 1 phosphorylates and regulates 14-3-3 binding to GEF-H1, a microtubule-localized Rho exchange factor. J Biol Chem 2004; 279: 18392-18400.

27 O'Toole TE, Bialkowska K, Li X, Fox JE. Tiam1 is recruited to beta1-integrin complexes by 14-3-3zeta where it mediates integrin-induced Rac1 activation and motility. J Cell Physiol 2011; 226: 2965-2978.

28 Hofbauer SW, Krenn PW, Ganghammer S, Asslaber D, Pichler U, Oberascher K et al. Tiam1/Rac1 signals contribute to the proliferation and chemoresistance, but not motility, of chronic lymphocytic leukemia cells. Blood 2014; 123: 2181-2188.

29 Kent OA, Sandi MJ, Burston HE, Brown KR, Rottapel R. An oncogenic KRAS transcription program activates the RHOGEF ARHGEF2 to mediate transformed phenotypes in pancreatic cancer. Oncotarget 2016; 8: 4484-4500.

30 Choi MY, Widhopf GF 2nd, Wu CC, Cui B, Lao F, Sadarangani A et al. Pre-clinical specificity and safety of UC-961, a first-in-class monoclonal antibody targeting ROR1. Clin Lymphoma Myeloma Leuk 2015; 15(Suppl): S167-S169.

(1) This work is licensed under a Creative Commons Attributioncc) NonCommercial-NoDerivs 4.0 International License. The images or other third party material in this article are included in the article's Creative Commons license, unless indicated otherwise in the credit line; if the material is not included under the Creative Commons license, users will need to obtain permission from the license holder to reproduce the material. To view a copy of this license, visit http:// creativecommons.org/licenses/by-nc-nd/4.0/

(c) The Author(s) 2017

Supplementary Information accompanies this paper on the Leukemia website (http://www.nature.com/leu) 